\title{
Making the kindest cut
}

A combination of the latest generation of optics and imaging technologies enables researchers to introduce and monitor targeted disruptions in live embryos, offering a powerful complement to genetic strategies for the study of embryo morphogenesis.

Genetic factors have a primary role in the morphological development of embryos, but there is evidence that the resulting tissue deformations also influence gene expression. Emmanuel Farge of the Curie Institute in Paris recently found that expression of one developmentally restricted gene, $t w i s t$, seems to be induced by cellular movements within the Drosophila melanogaster embryo (Farge, 2003). Emmanuel Beaurepaire, a collaborator of Farge's and optics specialist at the École Polytechnique in Palaiseau, France, was interested in exploring this process further, and the two men and their colleagues worked to develop a system to induce targeted lesions in embryos and track their impact on morphogenetic movements (Supatto et al. 2005).

Beaurepaire's team opted to use ultrashort near-infrared laser pulses for their targeted ablations. Preservation of the vitelline membrane is essential to embryonic survival during in vivo studies; by focusing their laser at a point beneath the membrane, they were able to make ablations without compromising the embryo's viability. Once the cuts were made, they tracked the outcome by a method known as particle imaging velocimetry (PIV). A commonly used technique in fluid dynamics, PIV uses algorithms to track individual particles within complex dynamic systems. By applying PIV after ablation, the researchers obtained quantitative velocity fields reflecting cell movements during development, and comparative analysis of these data revealed changes in deformation patterns resulting from specific manipulations.

Ablations targeted toward the middle of the embryo had a dramatic impact on lateral cell movements and on the formation of the cephalic furrow, which ultimately establishes the division between head and thorax.
Cells adjacent to the targeted region were not noticeably damaged, suggesting that the observed effects were primarily the result of the elimination of specific cells. The researchers next examined changes in twist expression in the anterior pole, which is thought to be induced by deformations that result from the migration of adjacent cells. Ablation of dorsal cells virtually eliminated these migrations, and twist expression at the anterior pole dropped dramatically.

The initial experiments were visualized by two-photon microscopy, the current gold standard among imaging methodologies. However, Beaurepaire and his colleagues also worked with a newer system, third-harmonic generation (THG) microscopy, which is just starting to find use in biological studies. In THG imaging samples are scanned with a focused, short-pulse laser. When the beam scans homogeneous portions of the sample, no signal is detected, owing to the optical properties of the beam; however, a clear signal is obtained when scanning a heterogeneous region, such as the interface between two structures. A key benefit of THG is that it uses a long-wavelength, mid-infrared beam (roughly 1,200 $\mathrm{nm}$ in this study) that allows deep penetration of a sample with minimized scattering or absorption. Together, these properties make THG well-suited for embryological imaging.

By combining THG and PIV, the team assembled striking images of the differences in cellular movement resulting from laser ablation (Fig. 1), and even the notoriously poor optics of the D. melanogaster embryo, which is highly opaque to visible light, posed no obstacle to visualization. Most importantly, according to Beaurepaire, no additional staining is necessary. "It's quite appropriate to image embryos, because you don't need to label anything. Of course, you can use GFP constructs— but if you want to study complex mutants, then it might be difficult to obtain GFP expression in that case, [and] this thirdharmonic microscopy might be very useful."

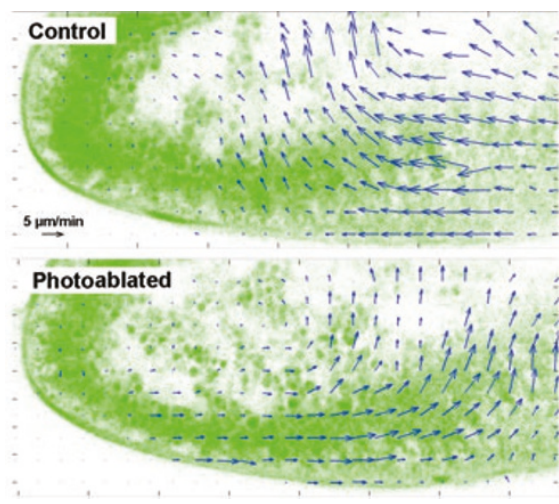

Figure 1 | Movements of cells and internal structures in D. melanogaster embryo early development as visualized using THG microscopy in an intact embryo (above), and in an embryo after intravital photoablation of specific dorsal tissues (below) resulting in altered movement of cells and internal structures at the anterior pole.

Beaurepaire believes that laser ablation is a technology whose time has come and suggests that the system could soon be simplified: "For example, it would be nice to have a dual laser source that would allow us to switch between high-energy pulses to do the ablation...and lower-energy pulses to do the imaging." Meanwhile, he and his colleagues are following up on the promising findings yielded by this first round of experiments. "Right now, we're interested in the application that is demonstrated in the paper," he says, "which is to study mechanosensitive gene expression during development. We think it's an interesting approach to do this because it relies on targeted non-genetic perturbations, so it can nicely complement genetic studies."

\section{Michael Eisenstein}

\section{RESEARCH PAPERS}

Supatto, W. et al. In vivo modulation of morphogenetic movements in Drosophila embryos with femtosecond laser pulses. Proc. Natl. Acad. Sci. USA 102, 1047 1052 (2005).

Farge, E. Mechanical induction of Twist in the Drosophila foregut/stomodeal primordium. Curr. Biol. 13, 1365-1377 (2003). 